\title{
Cirugia de Mohs: Aplicación de la técnica a neoplasias del pene
}

\author{
Cuevas J*, de Eusebio E**, Díez E**, Castiñeira I*. \\ *Servicio de Anatomía Patológica. **Servicio de Dermatología. Hospital Universitario de Guadalajara.
} Universidad de Alcalá de Henares. Madrid.

Actas Urol Esp. 2007;31(9):1076-1081

\section{RESUMEN}

CIRUGÍA DE MOHS: APLICACIÓN DE LA TÉCNICA A NEOPLASIAS DEL PENE

La cirugía de Mohs es una técnica quirúrgica que permite la extirpación en capas de tumores cutáneos con los más elevados porcentajes de curación. Al mismo tiempo permite el máximo ahorro de tejido sano. Todo ello es posible gracias a la supervisión microscópica que posibilita analizar el 100\% de los bordes tumorales de cada una de las capas y así poder guiar al cirujano en las sucesivas persistencias tumorales hasta la completa extirpación del tumor. La utilidad de la cirugía micrográfica de Mohs en el tratamiento de las neoplasias de pene tiene su justificación por el ahorro de tejido que permite. El carcinoma verrucoso de pene, constituye una indicación de cirugía de Mohs debido a la elevada tendencia a la recidiva local tras la cirugia convencional. Aunque más infrecuentes, otros tipos histológicos de tumores del pene en los que la cirugía micrográfica de Mohs puede ser de utilidad son: epitelioma basocelular, la enfermedad de Paget extramamaria, el melanoma in situ y el tumor de células granulosas.

Palabras clave: Cirugía micrográfica. Mohs. Pene.

\section{ABSTRACT}

MOHS MICROGRAPHIC SURGERY: APLICATION OF THIS TECHNIQUE TO PENILE NEOPLASMS

Mohs micrographic surgery is a surgical technique that allows the excision in successive layers of cutaneous malignancies with the higher cure rates. At the same time, this surgical technique offers the maximal preservation of normal tissue. That is possible because Mohs surgery provides the advantage of microscopically controlled tumor-free borders in each stage guiding the surgeon in the tumor persistence until the complete surgical excision. Mohs micrographic surgery is a precise treatment for penile neoplasms and its utility is justified because the removal of a substantial surgical margin of normal tissue is obviated. Mohs micrographic surgery is indicated in the treatment of penile verrucous carcinoma due to the significant risk of loco-regional recurrence after conventional surgery. Although infrequent, other penile neoplasms that can benefit from Mohs micrographic surgery are: basal cell carcinoma, extrammamary Paget's disease, in situ melanoma and granular cell tumor.

Keywords: Mohs. Micrographic surgery. Penis.

A lgunos tumores cutáneos localmente invasivos tienen, en función de su localización y tipo histológico, mayor frecuencia de recidivas tras la cirugía, incluso aunque el examen anatomopatológico de rutina realizado tras la extirpación haya indicado la ausencia de tumor en los márgenes de la extirpación. Cuando se realiza una extirpación simple por el método tradicional, la pieza se envía al laboratorio de Anatomía Patológica para confirmar que los márgenes quirúrgicos están libres, lo que se lleva a cabo seccionando la totalidad de la pieza mediante cortes verticales seriados cada $2-4 \mathrm{~mm}$. Esto es sufi- ciente para un tumor que presente una estructura compacta y unos márgenes bien definidos, pero esos 2-4 mm de tejido entre los cortes queda sin examinar. De esta manera, un determinado tumor que después de ser examinado con ésta técnica presente márgenes quirúrgicos libres, podría tener prolongaciones en estos intervalos que no se han examinado. Esto es debido al crecimiento asimétrico y agresivo de la neoplasia, que forma prolongaciones digitiformes de crecimiento lateral o en profundidad ${ }^{1}$. En muchas ocasiones, las células tumorales se propagan de forma poco perceptible siguiendo un patrón de 
invasión perineural, a través de hueso o cartílago, o siguiendo los denominados planos de fusión embrionaria. Dichos planos son teóricamente los que se originarian durante el desarrollo embrionario por la fusión de las superficies epiteliales ${ }^{2}$.

En la actualidad, existen diferentes técnicas para tratar con éxito los tumores que afectan a la piel y entre ellos al pene. Se ha utilizado curetaje, crioterapia, electrocirugía, cirugía convencional y radioterapia. La cifra máxima de curaciones con estos tratamientos es de 90-95\%, para los casos sin tratamiento previo, y $60-85 \%$ para las recidivas. Por este motivo se ha continuado la búsqueda de un método que fuera más efectivo que los anteriores. Todos ellos excepto la extirpación quirúrgica carecen de la capacidad de definir y confirmar los márgenes tumorales, debido a la imposibilidad de examinar histopatológicamente dichos márgenes.

Por ello la idea que ha primado en la búsqueda de una técnica ideal para el tratamiento de los tumores cutáneos ha sido añadir a ese futuro método, algo de lo que adolecía el resto: una visión microscópica que guiase al cirujano en el momento de la extirpación y que permitiese 2 hechos fundamentales: a) indicar de forma precisa y en el mismo momento operatorio la extensión del tumor y b) evitar grandes márgenes perilesionales de tejido sano innecesariamente extirpado $^{3}$. Estos objetivos son cumplidos en un porcentaje muy elevado de casos mediante la citada técnica de cirugía micrográfica de Mohs y tiene una gran aceptación en EEUU, donde cerca del $30 \%$ de todos los epiteliomas son intervenidos con esta modalidad quirúrgica y poco a poco va adquiriendo protagonismo en Europa.

\section{HISTORIA}

La idea de la extirpación de los tumores cutáneos bajo supervisión microscópica fue concebida por un estudiante de Medicina de la Universidad de Wisconsin, Frederick E Mohs (fallecido el 2 de Julio de 2002) cuando, de 1929 a 1934, realizaba prácticas en el Departamento de Zoología. Al estudiar comparativamente las diferentes respuestas del tejido normal y canceroso a la inyección intratumoral de sustancias destructivas, observó que al inyectar por error una solución de cloruro de zinc a una concentra- ción excesivamente alta (20\%), se produjo una necrosis tisular completa. Observó, al estudiarla al microscopio, que el tejido necrosado conservaba su arquitectura normal, habiendo sido fijado in situ y podía ser valorada igual que un tejido vivo extirpado y fijado posteriormente. A partir de esta observación, inició el desarrollo de una técnica que permitía la extirpación tumoral bajo control microscópico.

Inicialmente, el proceso, llamado quimiocirugía por su descubridor, consistía en la aplicación, la noche anterior a la intervención, de una pasta de cloruro de zinc sobre el tumor. Al día siguiente la intervención comenzaba por tomar una sección horizontal del tumor, sin dolor y sin sangrado. La muestra se colocaba de manera que los bordes y la cara inferior estuvieran situados en el mismo plano horizontal, para poder inspeccionar de esta manera todos los márgenes quirúrgicos. El marcaje meticuloso de los bordes del tumor y su orientación, permitían al cirujano volver a la localización exacta del resto tumoral. De nuevo se repetía el proceso de aplicación de la pasta de cloruro de zinc, y la obtención de una nueva sección transversal, limitada a la zona donde existía tumor, y así sucesivamente hasta que no quedaba ningún resto tumoral. Finalmente, la lesión se dejaba cicatrizar por segunda intención. Este proceso se describió como técnica de tejido fijado.

Aunque los porcentajes de curación obtenidos mediante esta técnica eran muy alentadores, existían varios inconvenientes para su utilización como técnica de rutina; el primero de ellos era que la aplicación de la pasta de cloruro de zinc durante toda la noche previa a la intervención era incómoda y mal tolerada por los pacientes; en segundo lugar, todo el proceso se alargaba demasiado en el tiempo, ya que, debido a la necesidad de aplicar nuevamente la pasta durante una noche antes de cada nueva sección, no se podía completar más de una etapa cada día y por último, la aplicación previa de esta pasta impedía la reconstrucción inmediata del defecto, ya que era necesario esperar a que se descostrara previamente para dejar tejido fresco que ya se podría cerrar.

En 1953, mientras filmaba una intervención, Mohs decidió utilizar ácido dicloroacético como 
agente hemostásico durante el curetaje inicial previo a la toma de la primera sección transversal, y anestesia local en lugar de cloruro de zinc, para acortar el proceso. De esta forma comprobó que la técnica era igualmente efectiva, pero simplificada y más rápida, y comenzó a aplicarla, con el nombre de técnica de tejido fresco, en tumores de pequeño tamaño, especialmente en aquellos que afectaban a la región palpebral, ya que en esta zona, la pasta de cloruro de zinc resultaba altamente irritante.

Posteriormente con la aparición de criostatos como forma mas cómoda para el corte en congelación de tejidos frescos y con la mejora de los anestésicos locales, la técnica de elección en la gran mayoría de casos es la de tejido fresco .

La técnica del tejido fresco, eliminaba todos los inconvenientes surgidos previamente, ya que no había molestia para el paciente, se podían realizar varias etapas e incluso finalizar la intervención en un solo día, y el defecto se podía cerrar de manera inmediata ${ }^{4}$.

\section{TÉCNICA DE LA CIRUGÍA MICROGRÁFICA DE MOHS}

La técnica de la cirugía de Mohs consiste en realizar de forma sistemática y ordenada una secuencia que en conjunto se denominan "pase" o "estadio". Al final de cada pase podremos saber si el tumor ha sido completamente extirpado o si por el contrario hay persistencia tumoral en cuyo caso será necesario realizar un nuevo pase y asî sucesivamente hasta completar la extirpación del tumor. El tejido es extirpado en "lonchas" finas paralelas a la superficie y orientado con formato horario siguiendo las agujas de un reloj y que se referencia en la pieza de extirpación y sincrónicamente en el paciente.
Además marcamos en la pieza zonas horarias con un código de colores que nos permite que podamos localizar la parte superior, inferior, derecha e izquierda. En el laboratorio, la pieza se procesa de forma que se puede estudiar al microscopio toda la periferia y su fondo en el mismo plano. En caso de que en alguna de las fracciones se observaran restos tumorales, se reintervendría de nuevo pero solo en esa zona. $\mathrm{El}$ proceso se repite hasta estar libre de tumor en todos los márgenes (Fig. 1).

El porcentaje de complicaciones quirúrgicas en la cirugía de Mohs, en la serie de Cook, es del $1,64 \%$ (22/1343) siendo en su mayoría problemas de hemostasia.

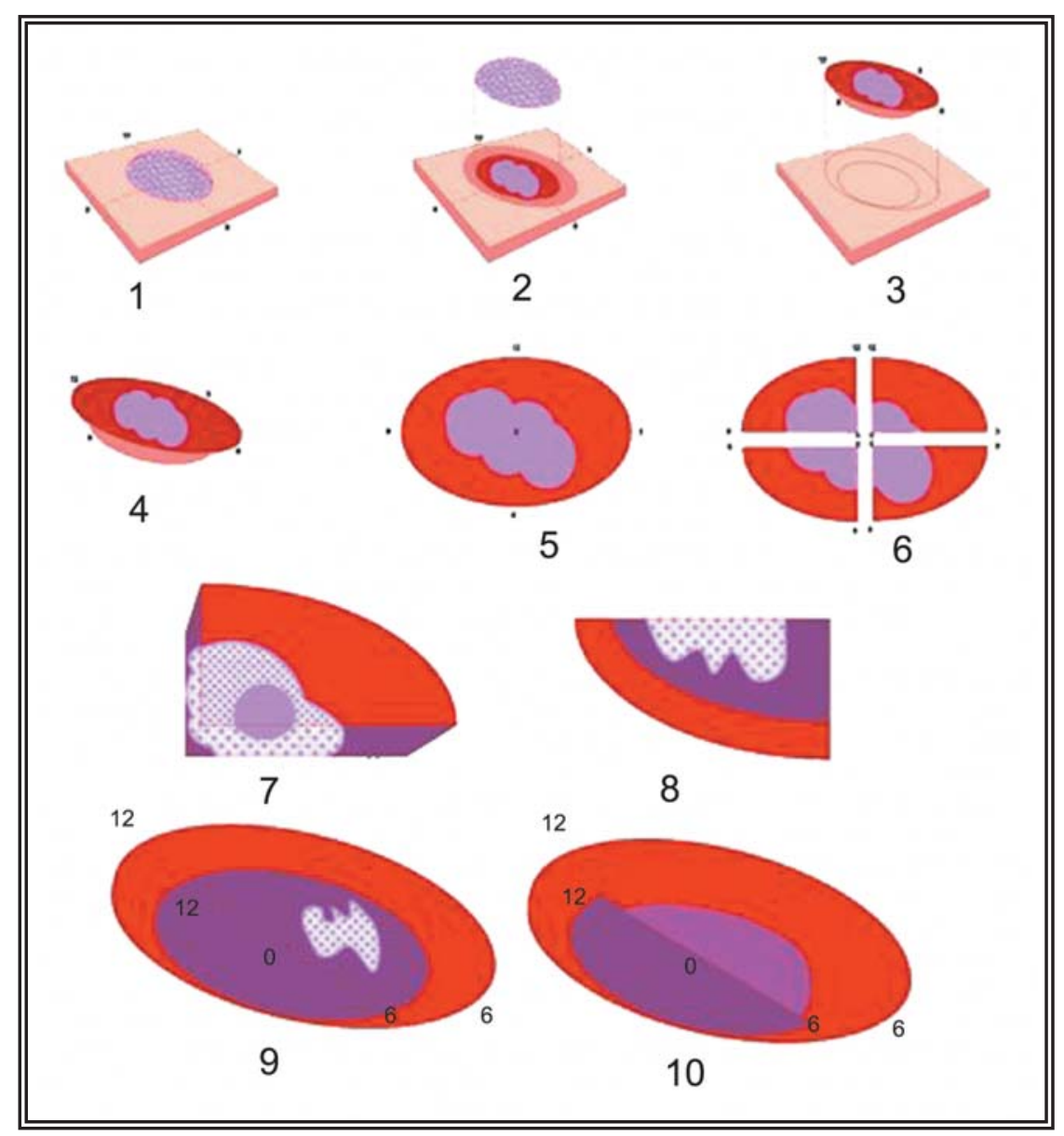

FIGURA 1. Técnica micrográfica de Mohs. Representación esquemática de los sucesivos pasos de esta técnica quirúrgica. 1, 2 y 3, extirpación de la neoplasia marcando su orientación; 4 y 5, marcaje de la pieza según un código horario (12, 3, 6 y 9 horas); 6, sección de la pieza en cuatro cuadrantes identificando el extremo correspondiente a las 3 y a las 12 horas; 7 y 8 estudio en congelación de cada uno de los cuadrantes valorando histológicamente la presencia o ausencia de neoplasia en cada uno de ellos; 9 y 10 representan los sucesivos pases en capas hasta que en el último (10) no quede neoplasia residual constatada microscópicamente. 


\section{INDICACIONES}

El hecho de que la CMM sea un procedimiento quirúrgico que logra mejores cifras de curaciones, no justifica que todos los tumores sean tratados con esta técnica dado que es costosa en tiempo, personal y laboratorio. La indicación de la cirugia micrográfica de Mohs (CMM ) se establece en función del tipo histológico de tumor, la localización y otras características como tamaño y recurrencia que vamos a revisar.

En cuanto el tipo histológico la CMM fue indicado en principio para el tratamiento del carcinoma basocelular (CB) y el carcinoma espinocelular (CE) y estas continúan siendo sus principales indicaciones. Sin embargo, como la técnica ha evolucionado, ha sido aplicada a otras neoplasias cutáneas, en muchos casos con un resultado superior al obtenido con los métodos publicados previamente.

El (CB) constituye la indicación más frecuente de CMM. Los subtipos histológicos con comportamiento biológico más agresivo son los que más se benefician de esta técnica como el infiltrativo, morfeiforme, metatípico o basoescamoso, y el superficial multicéntrico.

El Carcinoma Epidermoide (CE) es el segundo tipo histológico más frecuentemente tratado con CMM. Este tumor puede beneficiarse de la CMM únicamente en aquellas fases en las que no existe diseminación a los ganglios linfáticos regionales o metástasis a distancia. Estaría especialmente indicada en aquellos subtipos histológicos menos diferenciados o en aquellos que presenten invasión perineural. También el carcinoma verrucoso debido a su tendencia a la invasión profunda y a la elevada tasa de recidivas que desarrolla tras la cirugía convencional tiene indicación de Cirugía Micrográfica de Mohs ${ }^{5}$.

Otra indicación es el carácter recurrente de la neoplasia. La recidiva de un tumor tras una extirpación quirúrgica previa le proporciona un comportamiento biológico especial: el plano de despegamiento de los bordes se convierte en un plano de avance tumoral, la reconstrucción con un injerto o un colgajo proporciona múltiples vías de diseminación a través de unos tejidos seccionados y con menor resistencia a la invasión tumoral. Para ellos la cirugía de Mohs proporciona un mapa en 3 dimensiones que permite el seguimiento microscópico de las extensiones tumorales.
Otra indicación son aquellos tumores extirpados incompletamente ya que al carácter de diseminación subclínica no apreciable a primera vista se une el que se trate de una lesión intervenida previamente ${ }^{6}$.

La localización en áreas de importancia cosmética y funcional elevada o bien en aquellas en las que es importante la conservación de tejido sano como son la pirámide nasal, cantos externo e interno oculares, filtrum, región pre y postauricular y borde bermellón del labio en la cara.

Otras áreas de indicación de CMM es el área anogenital y especialmente el pene. La utilidad de la CMM en el tratamiento de los carcinomas de pene tiene su justificación por el ahorro de tejido que permite. En esta topografia se han obtenido tasas de curación del 100 por 100 en los de menos de $2 \mathrm{~cm}$ de diámetro, de 2 a $3 \mathrm{~cm}$ del 87 por 100 y de más de $3 \mathrm{~cm}$, del 50 por 100. La eritroplasia de Queirat que clínicamente se manifiesta como una placa roja, brillante y aterciopelada de la mucosa del glande o la cara interna del prepucio, y la papulosis bowenoide que se manifiesta como pápulas eritematosas son 2 variedades de Ca epidermoide in situ típicas del pene que se benefician del ahorro de tejido sano que conlleva el tratamiento con $\mathrm{CMM}^{7-9}$.

El carcinoma verrucoso, que supone aproximadamente el 5 a $16 \%$ de los tumores epidermoides en esta localización anatómica, constituye también una indicación de CMM debido a la elevada tendencia a la recidiva local tras la cirugía convencional ${ }^{10}$ (Fig. 2).

En la reconstrucción de esta zona la granulación espontánea es una buena alternativa en defectos de tamaños pequeños o medios y tiene la ventaja de producir mínimas cicatrices retráctiles, sobre todo en glande y surco balanoprepucial. Otra alternativa es la utilización de injertos cutáneos o de mucosa oral para la zona perimeatal.

Otros tipos histológicos de tumores que pueden desarrollarse en el pene con mucha menos frecuencia y para los que se ha descrito en casos aislados la utilidad de la CMM son por ejemplo el carcinoma basocelular ${ }^{11}$, la enfermedad de Paget extramamaria ${ }^{12-15}$ el melanoma in situ o el tumor de células granulosas ${ }^{16}$. En todas estas indicaciones la clave de la indicación de la CMM está en el ahorro de tejido sano que permite la utilización de la técnica ${ }^{17}$. 


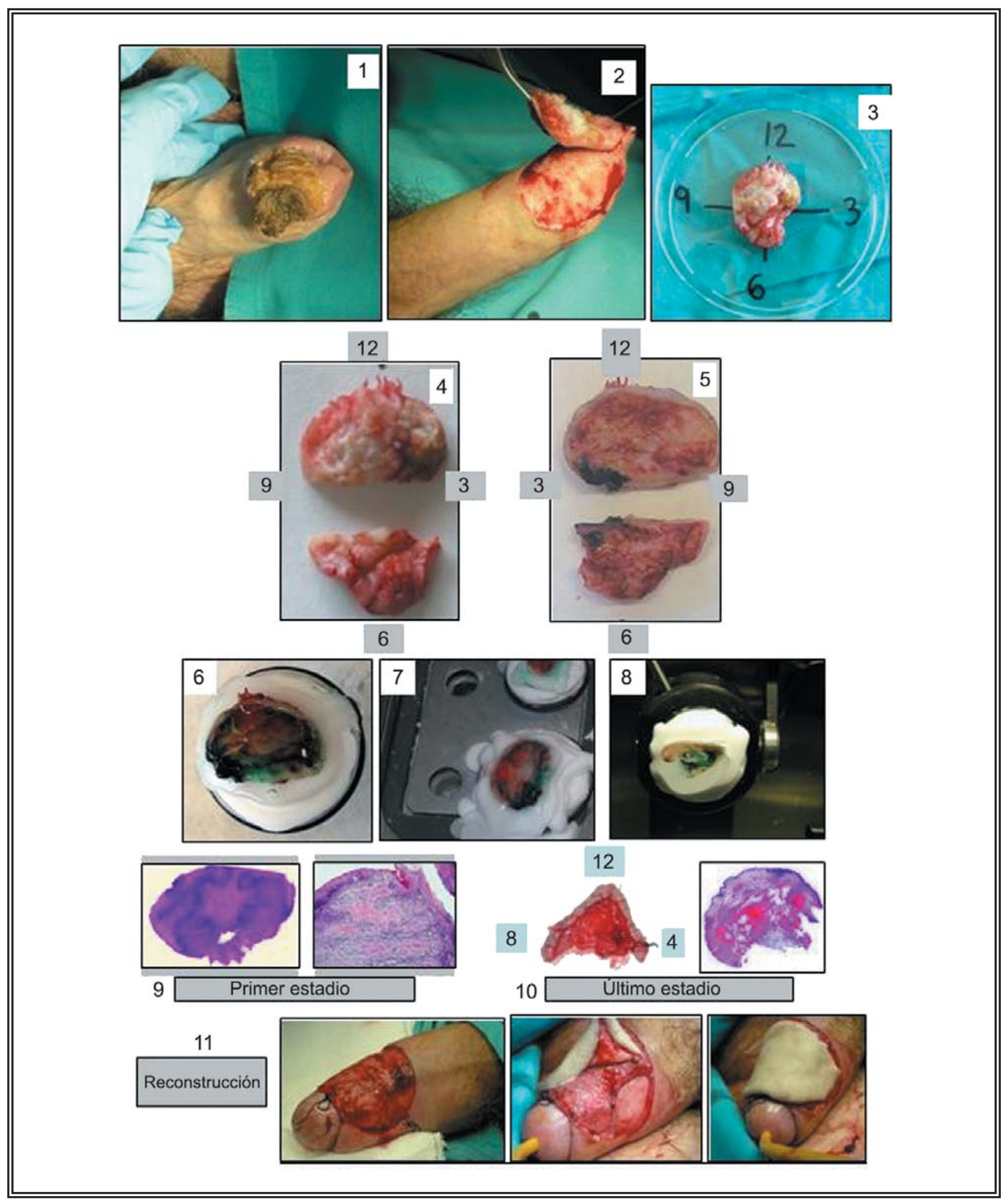

FIGURA 2. Procesamiento y resolución de un carcinoma verrucoso de pene con técnica de cirugía micrográfica de Mohs. 1 , imagen clinica de carcinoma verrucoso de pene; 2 , extirpación quirúrgica; 3 , orientación de la lesión extirpada en un placa de Petri orientada con un formato horario, marcando las 12, 3, 6 y 9 horas; 4 y 5, sección de las sucesivas capas; 6, 7 y 8, congelación de los distintos fragmentos de la pieza y sección en criostato; 9, imagen histológica panorámica del primer estadio con presencia de tumor; 10, imágenes macroscópica y microscópica del último estadio con ausencia de neoplasia; 11 , reconstrucción del lecho quirúrgico. 


\section{VENTAJAS E INCONVENIENTES DE LA CIRUGÍA MICROGRÁFICA DE MOHS EN EL TRATAMIENTO DEL CARCINOMA DE PENE}

La CMM tiene la ventaja de ser una técnica de gran efectividad en el tratamiento del cáncer de pene con la tasa de curaciones más elevada de todos los tratamientos propuestos. Además permite ahorrar un máximo de tejido sano ya que el control histológico de la CMM hace que las extirpaciones sean dirigidas, respetando el tejido sano perilesional.

La CMM implica un riesgo operatorio y postoperatorio muy bajo debido a que no suele necesitar anestesia general y el dolor, la hemorragia y la infección postoperatoria son muy poco frecuentes.

La reconstrucción del defecto suele producir un resultado cosmético y funcional mejor que otras técnicas debido a que se respeta al máximo el tejido sano perilesional.

El coste global de esta técnica en tumores complejos es menos elevado que otras alternativas debido a que evita reintervenciones ${ }^{10}$.

Las limitaciones o inconvenientes de la CMM son que requiere personal adiestrado tanto en las técnicas quirúrgicas como histopatológicas, así como pacientes colaboradores en intervenciones que pueden prolongarse varias horas.

A pesar de ello creemos que la opción de la CMM en el área genital está infrautilizada y tras una selección adecuada de pacientes candidatos puede ofrecer resultados más funcionales y especialmente puede evitar actitudes quirúrgicas más agresivas con las consiguientes secuelas físicas y psíquicas especialmente en pacientes con edades no avanzadas.

\section{REFERENCIAS}

1. Shiner DL, McCoy DK, Goldberg DJ, Vagner RF. Mohs Micrographic surgery. J Am Acad Dermatol 1998 Jul;39(1): 79-97.

2. Wentzell MJ, Robinson JK. Embryologic fusion planes and the spread of cutaneous carcinoma: a review and reassessment. J Dermatol Surg Oncol 1990;16(11):1000-1006.
3. Sánchez Conejo-Mir J. Cirugía Micrográfica de Mohs. En: Camacho F, de Dulanto F. Cirugía Dermatológica. Madrid: Aula Médica SA, 1995.p.261-264.

4. Brodland DG, Amonette R, Hanke IK, Robins P. The history and evolution of Mohs micrographic surgery. Dermatol Surg 2000;26(4):303-307.

5. Lang PG, Osguthorpe JD. Indications and imitations of Mohs micrographic surgery. Dermatol Clin 1989;7(4):627644.

6. Goldberg DJ, Schwartz RA. Basal cell carcinoma: cosmetic mutilation following incomplete excision. Can J Dermatol 1991; 17:574-578.

7. Mohs FE, Snow SN, Larson PO. Mohs micrographic surgery for penile tumours. Urol Clin North Am 1992;19(2):291-304.

8. Brown MD, Zachary CB, Grekin RC, Swanson NA. Genital tumours: their management by micrographic surgery. $\mathrm{J}$ Am Acad Dermatol 1988 Jan; 18(1 pt 1):115-122.

9. Brown MD, Zachary CB, Grekin RC, Swanson NA. Penile tumours: their management by Mohs micrographic surgery. J Dermatol Surg Oncol. 1987 Nov;13(11):1163-1167.

10. Muro Verdure I, Henries Manrique I, Sanz Jaka JP, Rekarte Barriola JA, Lluch Costa A. Verrucous carcinoma of the penis: local excision with the Mohs micrographic technique. Arch Esp Urol. 1996 Nov; 49(9):959-964.

11. Nguyen H., Saadat P, Bennet RG. Penile Basal Cell Carcinoma: Two cases treated with Mohs Micrgraphic Surgery and remarks on pathogenesis. Dermatol Surg 2006;32(1):135-144.

12. Hendi A, Brodland DG, Zitelli JA. Extramammary Paget's disease: surgical treatment with Mohs micrographic surgery. J Am Acad Dermatol. 2004 Nov;51(5):767-773.

13. O'Connor WJ et al. Comparison of Mohs micrographic surgery and wide excision for extramammary Paget's disease. Dermatol Surg. 2003 Jul;29(7):723-727.

14. Coldiron BM, Goldsmith BA, Robinson JK. Surgical treatment of extramammary Paget's disease. A report of six cases and a reexamination of Mohs micrographic surgery compared with conventional surgical excision. Cancer 1991 Feb 15;67(4):933-938.

15. Wagner RF Jr, Cottel WI. Treatment of extensive extramammary Paget disease of male genitalia with Mohs micrographic surgery. Urology. 1988 May;31(5):415-418

16. Gardner ES, Goldberg LH. Granular cell tumor treated with Mohs Micrographic Surgery: report of a case and review of the literature. Dermatol Surg 2001;27(8):772774.

17. Welch, Andermson LL, Grabski WJ. How many nonmelanoma skin cancers require Mohs micrographic surgery? Dermatol Surg 1996;22(8):711-713.

Correspondencia autor: Dr. J. Cuevas.

Servicio de Anatomía Patológica

Hospital Universitario de Guadalajara

Avda. Donantes de Sangre, s/n - 19002 Guadalajara

Tel.: 949209220

E-mail autor: jcuevas@sescam.org

Información artículo: Original - Patología del pene 